\title{
Molecular gas in Arp 94: Implications for intergalactic star formation
}

\author{
Ute Lisenfeld \\ Dept. Física Teórica y del Cosmos, Universidad Granada, Spain and Instituto de \\ Astrofísica de Andalucía (IAA/CSIC), Apdo. 3004, 18080 Granada, Spain \\ Carole Mundell \& James Allsopp \\ Astrophysics Research Institute, Liverpool John Moores University, Twelve Quays \\ House, Egerton Wharf, Birkenhead, CH41 1LD, U.K. \\ Eva Schinnerer \\ Max-Planck-Institut für Astronomie, Königstuhl 17, 69117 Heidelberg, Germany
}

\begin{abstract}
We present ${ }^{12} \mathrm{CO}(1-0)$ observations of the interacting galaxy system Arp 94, which contains the Seyfert galaxies NGC 3227 and NGC 3226 as well as the star-forming candidate dwarf galaxy J1023+1952. We mapped the CO distribution in J1023+1952 with the IRAM $30 \mathrm{~m}$ telescope and found molecular gas across the entire extent of the neutral hydrogen cloud - an area of about 9 by $6 \mathrm{kpc}$. The region where star formation (SF) takes place is restricted to a much smaller $(\sim 1.5$ by $3 \mathrm{kpc})$ region in the south where the narrow line width of the $\mathrm{CO}$ shows that the molecular gas is dynamically cold. Neither the molecular nor the total gas surface density in the $\mathrm{SF}$ region are significantly higher than in the rest of the object suggesting that an external trigger is causing the SF. The fact that $\mathrm{CO}$ is abundant and apparently a good tracer for the molecular gas in J1023+1952 indicates that its metallicity is relatively high and argues for a tidal origin of this object.
\end{abstract}

\section{Some background on Arp 94}

Arp 94 is an interacting Seyfert system (Mundell et al., 1995, 2004), consisting of the disturbed Seyfert galaxies NGC 3227, an SAB(s) pec barrred spiral, and its elliptical companion NGC 3226 (E2 pec). Neutral hydrogen (HI) imaging of the system (Mundell et al., 1995) revealed two gaseous tidal tails extending $\sim 100 \mathrm{kpc}$ north and south of NGC 3227, well-ordered gas in the disk of 

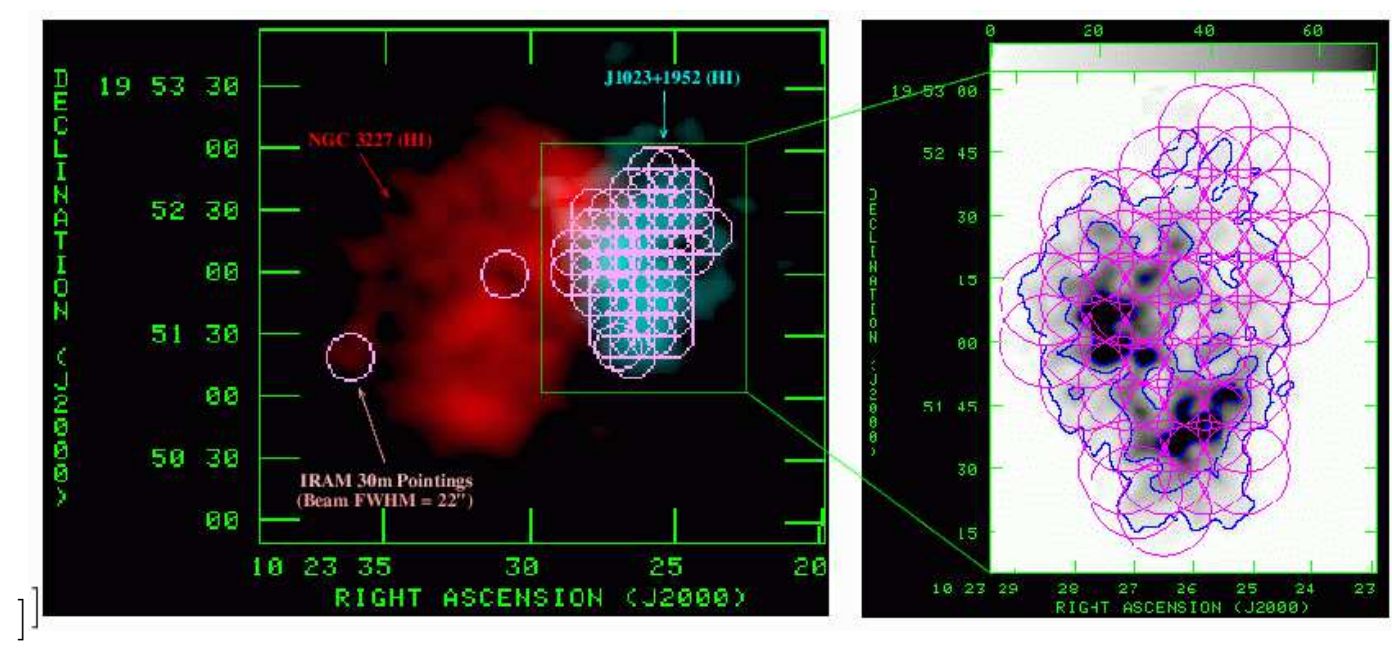

Fig. 1. Left: Location of IRAM $30 \mathrm{~m}$ pointings $\left(\mathrm{FWHM}=22^{\prime \prime}\right)$ overlaid on colour image of HI emission from spiral galaxy NGC 3227 (red) and J1023+1952 (blue). Right: Location of IRAM pointings on J1023+1952, overlaid over HI emission.

NGC 3227 and a massive HI cloud that lies apparently at the base of the northern tail and is close to, but physically and kinematically distinct from, the disk of NGC 3227. Mundell et al. (1995) suggested that this cloud (hereafter J1023+1952) might be a dwarf galaxy that is either pre-existing and being accreted by Arp 94, or a newly-created Tidal Dwarf Galaxy (TDG) forming from the tidal debris.

The subsequent discovery of a region of very blue star-forming knots embedded in a high HI column density ridge in the southern half of J1023+1952 (Mundell et al., 2004) confirmed its classification as a dwarf galaxy. The inferred star formation rate (SFR), from UV luminosities and $\mathrm{H} \alpha$ equivalent widths, of the knots suggested a starburst age less than 10 Myr. Near infrared imaging provided further evidence for the youth of J1023+1952, no additional embedded star formation (SF) or old stellar population were found (Mundell et al., 2004), a result confirmed by recent infrared imaging with Spitzer (Appleton et al., in prep.).

In this paper we present CO observations of J1023+1952, probing the distribution and kinematics of the molecular gas in order to better understand the origin of this object and how SF proceeds in it. A more detailed description of the observations, analysis and interpretation will be done in forthcoming publication. 


\section{CO observations and data analysis}

We observed the ${ }^{12} \mathrm{CO}(1-0)$ and ${ }^{12} \mathrm{CO}(2-1)$ (not shown here) lines in June 2004 with the IRAM 30-meter telescope at 115 and $230 \mathrm{GHz}$ with a standard setup. We used the wobbler with a throw of $200^{\prime \prime}$. The location of the individual pointing, spaced $10^{\prime \prime}$ apart within J1023+1952, plus two additional pointings within NGC 3227, are shown in Fig. 1. For the data reduction, we selected the observations taken during satisfactory weather conditions, summed the spectra over the individual positions and subtracted a constant continuum level. At the edge of the galactic disk of NGC 3227 the emission of this galaxy and $\mathrm{J} 1023+1952$ spatially overlaps in projection. It has been possible to disentangle the different kinematic components in the current ${ }^{12} \mathrm{CO}(1-0)$ data cube by the fitting of a double Gaussian line profile.

\section{Results}

$\mathrm{CO}$ was detected at practically all positions. In the nucleus and the outer disk of NGC 3227 strong CO emission was found. In Fig. 2 we show the observed spectra in J1023+1952, overlaid with HI spectra. The main results from the observations can be summarized as follows: (i) $\mathrm{CO}$ was detected everywhere in the HI cloud, and not just in the southern part where SF is taking place. The total extent of the $\mathrm{CO}$ emitting region is $\sim 6 \times 9 \mathrm{kpc}$. (ii) The line widths agree well with those of HI. (iii) The CO line widths vary over the cloud: They are narrow (FWZI of $30-40 \mathrm{~km} \mathrm{~s}^{-1}$ ) in the southern part coinciding with the SF region, and they are substantially broader in the rest of the cloud (FWZI of $>100 \mathrm{~km} \mathrm{~s}^{-1}$ ). The different line shapes are illustrated in Fig. 3 where typical spectra from these 3 regions are shown together with the corresponding Gaussian fits.

\section{Discussion}

\subsection{Triggering of the SF in the south}

The SF is restricted to a small region in the southern part. The absence of $\mathrm{SF}$ in the rest of the cloud is not due to a lack of molecular gas because $\mathrm{CO}$ has been found over the entire object. Neither is the surface density of the molecular gas the parameter determining the distribution of SF, because it is quite uniform over the cloud, with values between 3 and $6 \times 10^{20} \mathrm{~cm}^{-2}$. Furthermore, the highest molecular gas surface density does not coincide with 


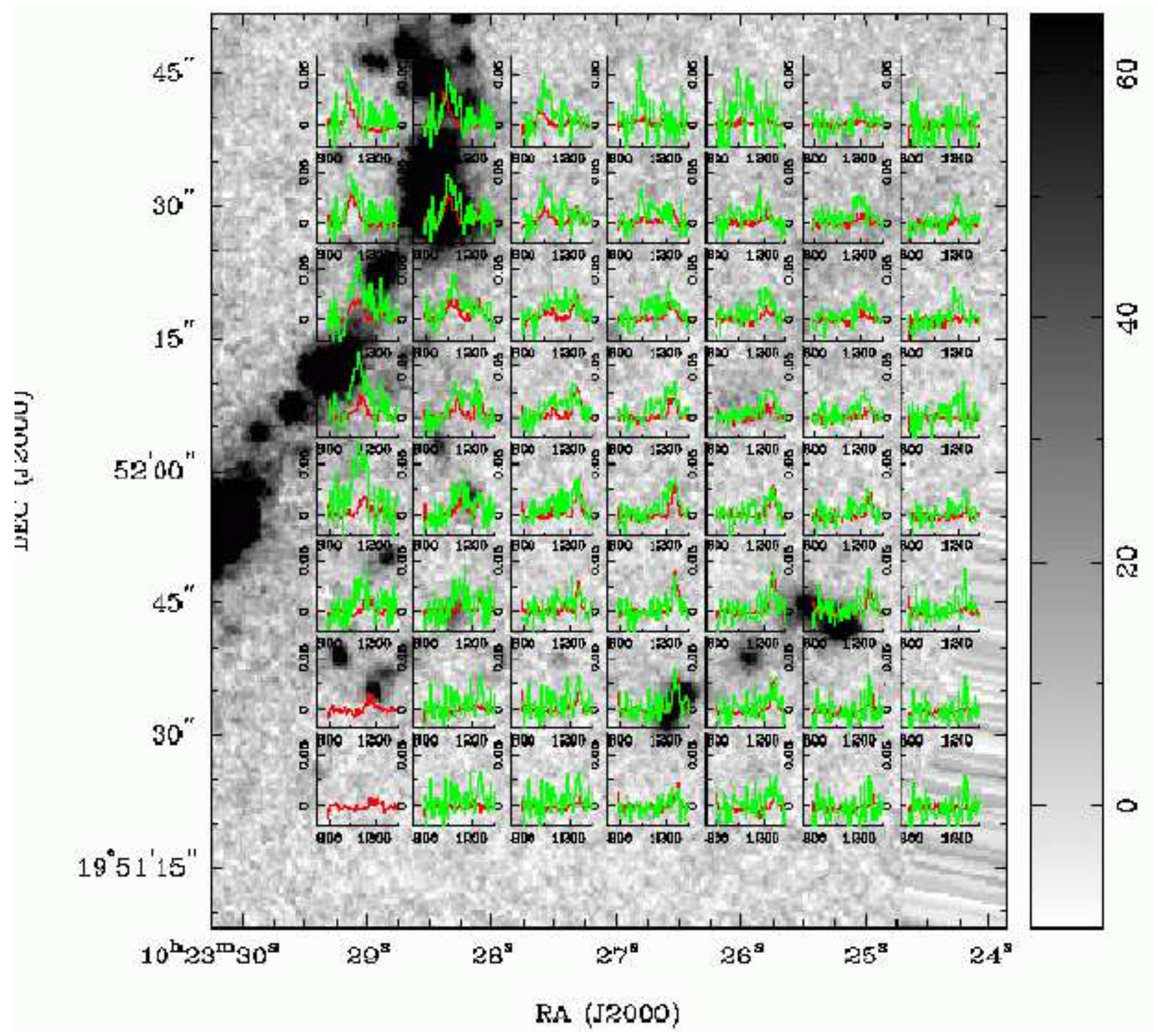

Fig. 2. Spectra of HI emission (red) and CO(1-0) emission (green) from J1023+1952 overlaid on $\mathrm{H} \alpha$ image from Mundell et al. (2004).

the SF region. The surface density of the total (atomic and molecular) gas is rather homogeneous as well with values ranging between 1.5 and $4 \times 10^{21}$ $\mathrm{cm}^{-2}$. Although the gas surface density in the SF region is close to the highest values, other places along the $\mathrm{HI}$ ridge have similarly high values, so that high gas surface density alone cannot be an explanation for SF occuring in just one restricted area.

The only noticable difference in the gas properties is the narrower line width in the region where SF takes place in comparison to the rest of the cloud, indicating that the gas is dynamically cooler here. The SF is triggered only in the dynamically-cold molecular gas in the south of the cloud, showing that overall gas-richness is not a sufficient condition for extragalactic star forma- 

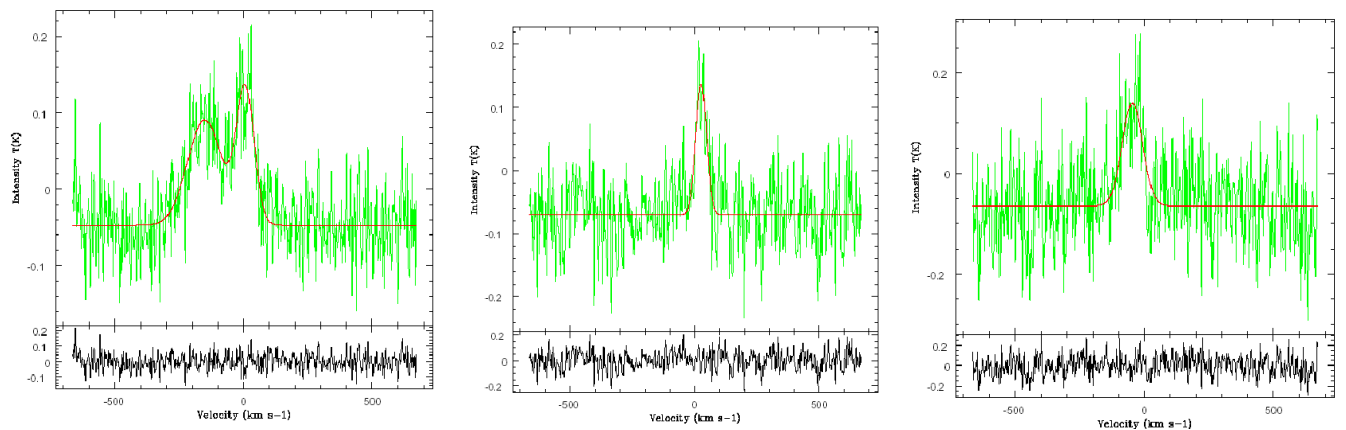

Fig. 3. CO spectra together with gaussian fittings for 3 different regions: Left: Region along the HI ridge close to NGC 3227 (around position RA 10:23:28.0, DEC 19:52:00). Middle: Region in the south with recent SF (around position RA 10:23:25.5, DEC 19:51:40). Right: Region towards the north-west, with low HI emission (around position RA 10:23:25.5, DEC 19:52:20).

tion. We suggest that the cold ridge and its associated SF are likely externally triggered by the tidal interaction.

\subsection{Nature of J1023+1953}

It has been an open question whether J1023+1953 is a preexisting dwarf galaxy, involved in the interaction, or an object created out of tidal debris stripped from the disk of NGC 3227, a candidate TDG. One property that distinguishes dwarf galaxies from TDGs is their metallicity. Since the latter are made from recycled gas, their metallicities are close to those of the parent galaxies. Therefore, TDGs do not follow the magnitude-luminosity relation found for other dwarf galaxies, and their metallicities lie in a narrow range of $12+\log (\mathrm{O} / \mathrm{H})=8.3-8.6$ (Duc et al., 2000).

Unfortunately, the metallicity in J1023+1953 has not been measured yet. However, the detection of large quantities of CO argues for a high metallicity. The conservative estimate of J1023+1952's total brightness $M_{B} \sim-15.5$, assuming an extinction of $A_{V}=2 \mathrm{mag}$, (Mundell et al 2004) would predict a metallicity of $12+\log (0 / \mathrm{H}) \sim 8$. At these low metallicities the detection rate of dwarf in $\mathrm{CO}$ is very low (e.g. Lerov et al. 2005), indicating that CO is no longer a good tracer of the molecular gas content. Thus, the fact that we detect abundant molecular gas, as usually found in TDGs (Braine et al., 2001), suggests that the metallicity is close to the values found in spirals and strongly argues for a tidal origin of J1023+1953.

\section{References}

Braine, J., Duc, P.-A., Lisenfeld, U., et al., 2001, A\&A, 378, 51 
Duc, P.-A., Brinks, E., Springel, V., et al., 2000, AJ, 120, 1238

Leroy, A., Bolatto, A. D., Simon, J. D, Blitz, 2005, ApJ, 625, 763

Mundell, C.G., Pedlar, A., Axon, D.J., Meaburn, J., Unger, S.W., 1995, MNRAS 277, 641

Mundell, C.G., James, P.A., Loiseau, N., Schinnerer, E., Forbes, D. A., 2004, ApJ, 614, 648 